\title{
Nucleon, Delta and Omega excited state spectra at three pion mass values
}

\section{John Bulava}

NIC, DESY, Platanenallee 6, D-15738, Zeuthen, Germany

Email: john.bulava@desy.de

\section{Robert G. Edwards, Bálint Joó, David G. Richards}

Thomas Jefferson National Accelerator Facility, Newport News, VA 23606, USA

Email: edwardsajlab.org, bjoo@jlab.org, dgrejlab.org

\section{Eric Engelson}

In-Depth Engineering Corp., 11350 Random Hills Road, Fairfax, VA 22030, USA

Email: engelson@gmail.com

\section{Huey-Wen Lin}

Department of Physics, University of Washington, Seattle, WA 98195, USA

Email: hwlin@phys.washington.edu

\section{Colin Morningstar}

Department of Physics, Carnegie Mellon University,Pittsburgh, PA 15213, USA

Email: colin_morningstarecmu.edu

\section{Stephen J. Wallace*}

Department of Physics, University of Maryland, College Park, MD 20742, USA

E-mail: stevewaleumd. edu

\begin{abstract}
The energies of the excited states of the Nucleon, $\Delta$ and $\Omega$ are computed in lattice QCD, using two light quarks and one strange quark on anisotropic lattices. The calculations are performed at three values of the pion mass: $m_{\pi}=392(4), 438(3)$ and 521(3) MeV. We employ the variational method with a basis of about ten interpolating operators enabling six energies to be distinguished clearly in each irreducible representation of the octahedral group. We compare our calculations of nucleon excited states with the low-lying experimental spectrum. There is reasonable agreement for the pattern of states.
\end{abstract}

The XXVIII International Symposium on Lattice Field Theory

June 14-19, 2010

Villasimius, Sardinia Italy

\footnotetext{
* Speaker.
} 


\section{Introduction}

The goal of determining the spectrum of hadron masses from lattice QCD is addressed in this work by calculations of the excited state spectrum of the nucleon, $\Delta$ and $\Omega$ using anisotropic lattices.[1] Earlier excited-baryon analyses were based on quenched QCD [2] and two-light-flavor $\left(N_{f}=2\right)$ QCD [3]. In this work we use ensembles of gauge configurations developed in Ref. [4] for $N_{f}=2+1$ QCD with two dynamical light quarks and one strange quark. The lattices are $16^{3} \times 128$ with spatial and temporal lattice spacings $a_{s}=0.122 \mathrm{fm}$ and $a_{t}=0.035 \mathrm{fm}$.

For families of particles with given isospin and strangeness, spectra are calculated in the six double-valued irreducible representations (irreps) of the octahedral group. There are three irreps for even-parity that are labeled with a $g$ subscript (gerade) and three for odd-parity that are labeled with a $u$ subscript (ungerade). They are: $G_{1 g}, H_{g}, G_{2 g}, G_{1 u}, H_{u}$ and $G_{2 u}$.

Sets of seven to eleven three-quark operators are used in each irrep and the variational method [5, 6] is used to extract energies of six states. Most operators incorporate gauge-covariant displacements of the quarks relative to one another in order to obtain nontrivial shapes. [7] The recently developed "distillation" method [8] is used for quark smearing. We start with a large set of operators in each irrep and then "prune" them to sets of about 10 that have the lowest condition numbers. That yields sets of approximately linearly-independent operators that are suitable for calculations based on diagonalizing a matrix of correlation functions.

\section{Results}

A detailed presentation of all of our results is given in Ref. [1]. Here we present selected results for the nucleon and $\Delta$ excited states.

Plots of the nucleon effective energies, calculated as

$$
E_{\mathrm{eff}}(t)=\frac{1}{2} \ln \left(\frac{\tilde{\lambda}(t-1)}{\widetilde{\lambda}(t+1)}\right),
$$

where $\tilde{\lambda}(t)$ is an eigenvalue of the generalized eigenvalue problem, are shown in Figure 1 for the $G_{1 g}$ and $G_{1 u}$ irreps. These plots show the values of $E_{\text {eff }}$ obtained from Eq. (2.1) as vertical bars and $E_{\text {eff }}$ calculated using the fit function,

$$
\lambda_{\text {fit }}(t)=(1-A) e^{-E\left(t-t_{0}\right)}+A e^{-E^{\prime}\left(t-t_{0}\right)},
$$

in place of $\tilde{\lambda}(t)$ in Eq. (2.1) as curved dashed lines. Comparison of the curved dashed lines with the bars from the lattice ensembles shows the usefulness of two-exponential fits. The term $A e^{-E^{\prime}\left(t-t_{0}\right)}$ models the contributions of higher energy states at early times allowing the exponential term $(1-$ A) $e^{-E\left(t-t_{0}\right)}$ to be determined over a larger fit window $\left(t_{i}, t_{f}\right)$ than would be possible using a single exponential. Fit energy $E$ and uncertainty of the fit energy, $\sigma$, are shown by dashed horizontal lines at $E+\sigma$ and $E-\sigma$ extending over the fit window. Note that the statistics allow credible determinations of six energy levels in each irrep. This provides evidence that quark smearing based on "distillation" is effective with regard to suppressing high-frequency fluctuations in the gauge ensembles. 
The energies obtained from the $G_{1 g}$ and $G_{1 u}$ effective mass plots of Fig. 1 are shown as boxes extending from $E-\sigma$ to $E+\sigma$ in Fig. 2. We show nucleon energies that are obtained in the same manner as shown in Fig. 1 for all irreps of the octahedral group and three pion masses. Experimental spectra are shown to the left of lattice energies for the spins and parities that have subductions to the lattice irreps. Lattice and experimental spectra are shown in Fig. 3 for the $\Delta$ family. See Ref. [1] for the $\Omega$ spectra.

In the nucleon spectra, there is good evidence for a spin $\frac{5}{2}^{-}$state. We find nearly degenerate $H_{u}$ and $G_{2 u}$ partner states, which is the signature of spin $\frac{5}{2}^{-}$. However, other spins are difficult to identify because there are many nearly-degenerate states, within uncertainties. It is a near-term goal within the collaboration to address spin identification by using operators that are subduced from continuum spins.

Our lattice spectra show scant evidence for multiparticle states even though many energies lie above the relevant thresholds. This is probably because single-hadron operators are used. There is an inference for a multiparticle contribution in that we find four low-lying states in $H_{u}$ while there are three low-lying experimental states that have subductions to $H_{u}$. There is a threshold for a multiparticle state in the same energy range. Our lattice results agree with the experimental pattern if one of the four low-lying $H_{u}$ states is multiparticle. However, we cannot identify the multiparticle states in the spectrum. It is a near-term goal within the collaboration to incorporate multiparticle operators that couple directly to such states.

Some lattice states appear to be "squeezed" by the small lattice volume used. They show up at higher energies than would be the case in a larger volume. The $G_{2}$ states require partner states in other irreps, such as $H$, in order to realize all the magnetic substates for a given spin. The partners should be close to the same energy. However, in the $\Delta$ spectra of Fig. 3 we find $G_{2}$ states at high energies without suitable partners being evident. Possibly they have been "squeezed". It is also a goal to perform calculations of spectra at larger volumes.

Although we do not attempt to extrapolate energies to $m_{\pi}=140 \mathrm{MeV}$, it is evident from Figs. 2 and 3 that the lowest-energy states on the lattice tend toward the energies of the physical resonances as the pion mass decreases. Decreasing the pion mass is an obvious goal but we recognize that it entails a more complex analysis for excited states that can decay.

\section{Summary}

This work represents a milestone in our long-term research program aimed at determining the spectra of baryons in QCD. It provides the first spectrum for $\mathrm{N}, \Delta$ and $\Omega$ baryons based on $N_{f}=$ $2+1$ QCD with high statistics. A large number of baryon operators is used to calculate matrices of correlation functions. They are analyzed using the variational method with fixed eigenvectors. The analysis provides spectra at three pion masses: $m_{\pi}=392$ (4) MeV, 438(3) MeV and 521(3) MeV.

The lattice volume and pion masses used give considerably higher energies than the experimental resonance energies. However, there is reasonable agreement of the overall pattern of lattice and experimental states. One exception is that almost all $\Delta$ states in the $G_{2}$ irrep are too high. That may be caused by a volume that is too small for highly excited states. 


\section{Acknowledgments}

This work was done using the Chroma software suite [9] on clusters at Jefferson Laboratory and the Fermi National Accelerator Laboratory using time awarded under the USQCD Initiative. JB and CM acknowledge support from U.S. National Science Foundation Award PHY-0653315. EE and SW acknowledge support from U.S. Department of Energy contract DE-FG02-93ER-40762. HL acknowledges support from U.S. Department of Energy contract DE-FG03-97ER4014. BJ, RE and DR acknowledge support from U.S. Department of Energy contract DE-AC05-060R23177, under which Jefferson Science Associates, LLC, manages and operates Jefferson Laboratory. BJ and RE acknowledge support under U.S. Dept. of Energy SciDAC contracts DE-FC02-06ER41440 and DE-FC02-06ER41449. The U.S. Government retains a non-exclusive, paid-up, irrevocable, world-wide license to publish or reproduce this manuscript for U.S. Government purposes.

\section{References}

[1] J. M. Bulava et al., Nucleon, $\Delta$ and $\Omega$ excited state spectra in $N_{f}=2+1$ lattice QCD, Phys. Rev. $D$ 82, 014507 (2010) [arXiv: 1004 . 5072].

[2] S. Basak et al., Lattice QCD determination of patterns of excited baryon states, Phys. Rev. D 76, 074504 (2007) [arXiv:0 709.0008$].$

[3] J. M. Bulava et al. , Excited State Nucleon Spectrum with Two Flavors of Dynamical Fermions, Phys. Rev. D 79034505 (2009), [arXiv: 0901.0027$].$

[4] H-W. Lin et al., First results from 2+1 dynamical quark flavors on an anisotropic lattice: light-hadron spectroscopy and setting the strange-quark mass, Phys. Rev. D 79, 034502 (2009) [arXiv:0810.3588].

[5] C. Michael, Adjoint sources in lattice gauge theory, Nucl. Phys. B 259, 58 (1985).

[6] M. Lüscher and U. Wolff, How to calculate the elastic scattering matrix in two-dimensional quantum field theories by numerical simulation, Nucl. Phys. B339, 222 (1990).

[7] S. Basak et al., Group-theoretical construction of extended baryon operators in lattice QCD, Phys. Rev. D 72094506 (2005) [arXiv: hep-lat/0506029].

[8] M. Peardon et al., Novel quark-field creation operator construction for hadronic physics in lattice QCD, Phys. Rev. D 80, 054506 (2009) [arXiv:0905.2160].

[9] R. G. Edwards and B. Joó, The Chroma Software System for Lattice QCD, Nucl. Phys. B. Proc. Suppl. 140, 832 (2005) [hep-lat/ 0409003 ]. 

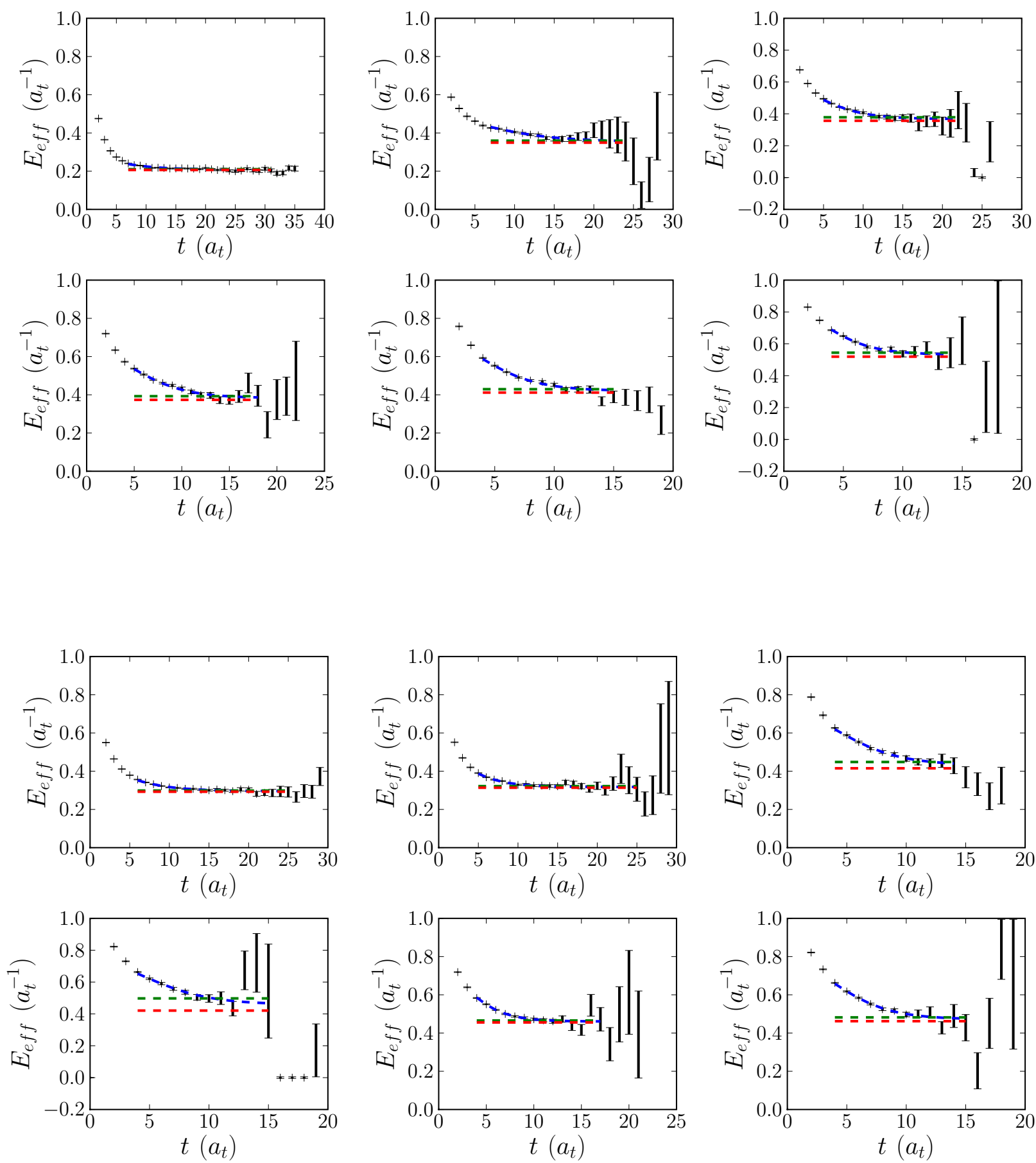

Figure 1: Nucleon $G_{1 g}$ effective energies are shown for the lowest states in the upper six graphs. The effective energy increases from left to right along the first row and continues to increase from left to right along the second row. The lower six graphs show nucleon $G_{1 u}$ effective energies increasing in the same pattern. Calculations are for $m_{\pi}=392(4) \mathrm{MeV}$. Vertical bars show the effective energy and the curved dashed line shows the effective energy calculated from the fit function. Horizontal dashed lines show the fit results for $E \pm \sigma$ and their extent shows the fitting interval $\left(t_{i}, t_{f}\right)$. 

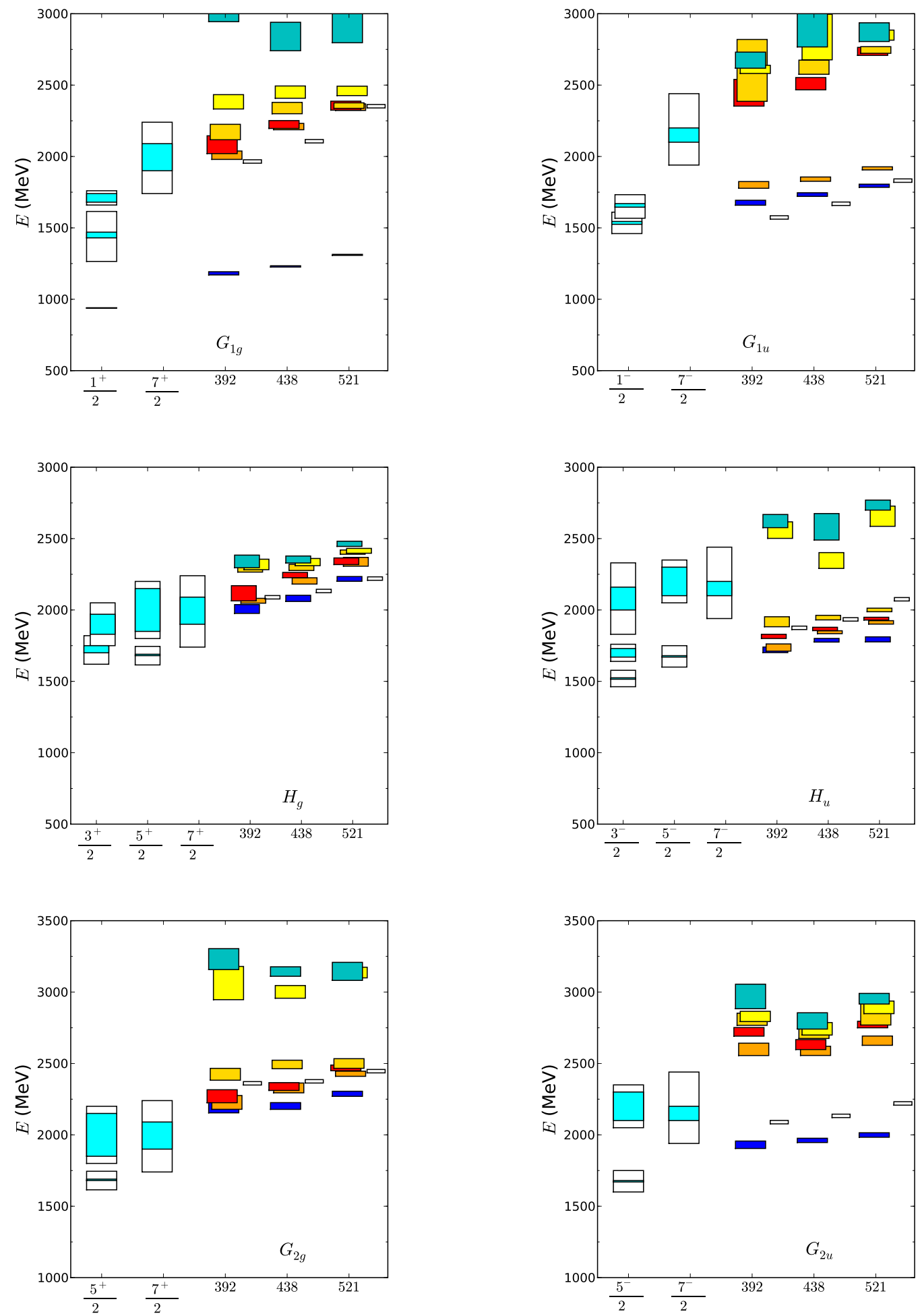

Figure 2: Spectra for isospin $\frac{1}{2}$ (nucleon family) at three values of $m_{\pi}$ in each irrep of the cubic group are compared with experimental spectra. Columns labeled by $m_{\pi}=392,438$ and $521 \mathrm{MeV}$ show lattice spectra. The $G_{1 g}$ and $G_{1 u}$ spectra in the $m_{\pi}=392 \mathrm{MeV}$ column are obtained from the plots of Fig. 1. Boxes extend from $E-\sigma$ to $E+\sigma$. Two, three and four-star experimental resonances are shown to the left of lattice spectra in columns labeled by their $J^{P}$ values. Each $J^{P}$ value listed has a subduction to the lattice irrep shown. Each box for an experimental resonance has height equal to the full decay width and an inner box (color aqua) showing the uncertainty in the Breit-Wigner energy. Open boxes show the thresholds for multiparticle states. 

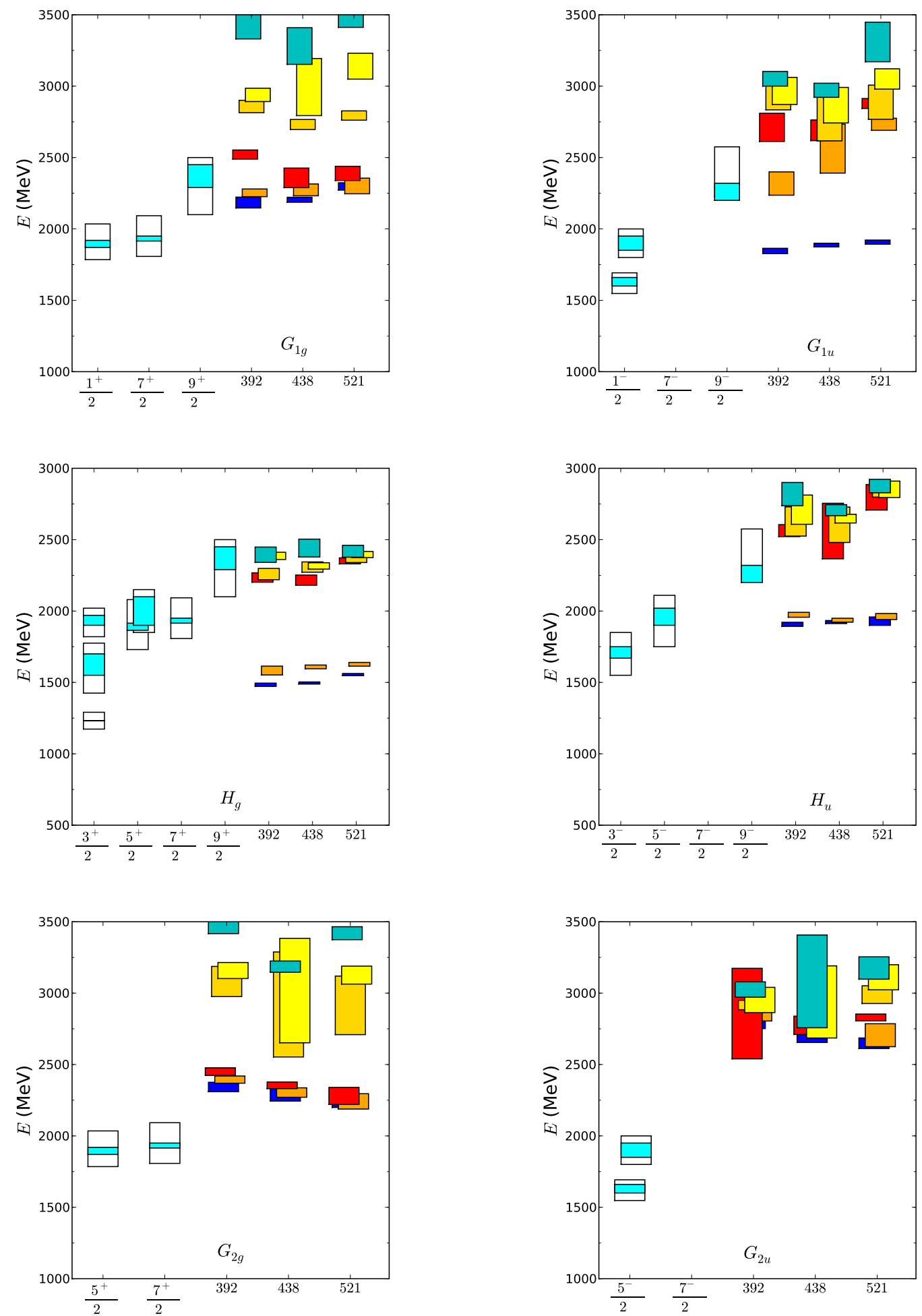

Figure 3: Spectra for isospin $\frac{3}{2}$ ( $\Delta$ family) at three values of $m_{\pi}$ in each irrep of the cubic group are compared with experimental spectra. Columns labeled by $m_{\pi}=392,438$ and $521 \mathrm{MeV}$ show lattice spectra. Boxes extend from $E-\sigma$ to $E+\sigma$. Two, three and four-star experimental resonances are shown to the left of lattice spectra in columns labeled by their $J^{P}$ values. Each $J^{P}$ value listed has a subduction to the lattice irrep shown. Each box for an experimental resonance has height equal to the full decay width and an inner box (color aqua) showing the uncertainty in the Breit-Wigner energy. 